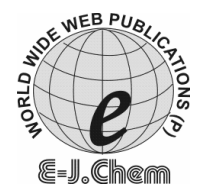

http://www.e-journals.net
ISSN: 0973-4945; CODEN ECJHAO

E-Journal of Chemistry 2010, 7(2), 514-516

\title{
Synthesis and Anti-Inflammatory Activity of Pyrazolines
}

\author{
B. RAMESH ${ }^{*}$ and T.SUMANA \\ Department of Pharmaceutical Chemistry, \\ Sree Siddaganga College of Pharmacy, \\ B.H. Road, Tumkur-572102. Karnataka, India. \\ rameshbbatta@gmail.com
}

Received 19 August 2009; Accepted 10 October 2009

\begin{abstract}
Some new pyrazoline derivatives were synthesized by reacting chalcones of 2-acetyl thiophene with phenyl hydrazine hydrochloride in the presence of alcohol. The synthesized compounds were identified by spectral data and screened for anti-inflammatory activity. Some of these compounds showed moderate to considerable anti-inflammatory activity.
\end{abstract}

Keywords: Synthesis, Pyrazolines, Anti-inflammatory activity.

\section{Introduction}

Compounds with pyrazoline structures are known to possess antimicrobial ${ }^{1-3}$, antiinflammatory ${ }^{4}$, antidepressant ${ }^{5,6}$, anti-tubercular ${ }^{7}$ activities. In the present study some new pyrazoline derivatives (1-5) have been synthesized by the reaction of chalcones of 2-acetyl thiophene and phenyl hydrazine hydrochloride. The structures of the various synthesized compounds are assigned on the basis of elemental analysis, IR and ${ }^{1} \mathrm{H}$ NMR spectral data. These compounds were also screened for their anti-inflammatory activity.

\section{Experimental}

Melting points were determined on a capillary melting point apparatus and are uncorrected.

${ }^{1} \mathrm{H}$ NMR spectra was recorded in the indicated solvent on Bruker WM $400 \mathrm{MHz}$ spectrometer with TMS as internal standard. Infrared spectra were recorded in $\mathrm{KBr}$ on Perkin-Elmer AC-1 spectrophotometer. Microanalyses were performed on Carlo Erba EA1108 element analyzer and were within the $\pm 0.5 \%$ of the theoretical values. Column chromatography was performed on silica gel (Merck, 60-120 mesh).

General procedure for the preparation of 2-pyrazolines (1-5)

A chalcone of 2-acetyl thiophene $(0.001 \mathrm{~mol})$ dissolved in $20 \mathrm{~mL}$ of ethanol and phenyl hydrazine hydrochloride $(500 \mathrm{mg}$ ) was added to it. To this mixture $0.3 \mathrm{~mL}$ of pyridine was added drop wise at room temperature. After that the mixture was refluxed for 5-6 hours and 
the solvent was evaporated completely. The reaction mixture was poured into ice-cold water and the solid mass that separated out was filtered, dried and purified by column chromatography with ethyl acetate/ hexane and recrystallized from chloroform.<smiles>[R]C=CC(=O)c1ccc(O)s1</smiles>

(1-5)

(1)<smiles>c1ccsc1</smiles>

(2)<smiles>Cc1ccc(N(C)C)cc1</smiles>

(3)<smiles>Cc1ccc(Cl)cc1</smiles>

(4)<smiles>Clc1ccc(Cl)cc1</smiles>

(5)<smiles>Fc1ccc(F)cc1</smiles>

Scheme 1. Synthesis of some new 2-pyrazoline derivatives

Table 1. Physical data of compounds (1-5)

\begin{tabular}{|c|c|c|c|c|c|c|c|c|c|}
\hline \multirow{3}{*}{ Compound } & \multirow{3}{*}{ M.F. } & \multirow{3}{*}{$\begin{array}{c}\text { M.P, } \\
{ }^{\circ} \mathrm{C}\end{array}$} & \multirow{3}{*}{$\begin{array}{c}\text { Yield, } \\
\%\end{array}$} & \multicolumn{6}{|c|}{ Elemental analyses, \% } \\
\hline & & & & \multicolumn{2}{|c|}{$\mathrm{C}$} & \multicolumn{2}{|c|}{$\mathrm{H}$} & \multicolumn{2}{|c|}{$\mathrm{N}$} \\
\hline & & & & Calcd & Found & Calcd & Found & Calcd & Found \\
\hline 1 & $\mathrm{C}_{17} \mathrm{H}_{12} \mathrm{~N}_{2} \mathrm{~S}_{2}$ & 210 & 71 & 66.23 & 66.12 & 3.89 & 3.81 & 9.09 & 9.06 \\
\hline 2 & $\mathrm{C}_{21} \mathrm{H}_{19} \mathrm{~N}_{3} \mathrm{~S}$ & 137 & 74 & 72.07 & 71.86 & 5.80 & 5.61 & 12.61 & 12.54 \\
\hline 3 & $\mathrm{C}_{19} \mathrm{H}_{13} \mathrm{~N}_{2} \mathrm{SCl}$ & 239 & 74 & 67.65 & 67.64 & 3.8 & 3.74 & 8.30 & 8.28 \\
\hline 4 & $\mathrm{C}_{19} \mathrm{H}_{12} \mathrm{~N}_{2} \mathrm{SCl}_{2}$ & 246 & 73 & 60.96 & 60.81 & 3.2 & 3.04 & 7.48 & 7.42 \\
\hline 5 & $\mathrm{C}_{19} \mathrm{H}_{13} \mathrm{~N}_{2} \mathrm{SF}$ & 227 & 68 & 71.47 & 71.42 & 4.07 & 4.04 & 8.77 & 8.74 \\
\hline
\end{tabular}

Table 2. Spectral data of the compounds (1-5).

\begin{tabular}{cll}
\hline Compound & \multicolumn{1}{c}{$\mathrm{IR}\left(\mathrm{KBr}, \mathrm{cm}^{-1}\right)$} & \multicolumn{1}{c}{${ }^{1} \mathrm{H} \mathrm{NMR}\left(\mathrm{CDCl}_{3}, \mathrm{ppm}\right)$} \\
\hline $\mathbf{1}$ & $1594(\mathrm{C}=\mathrm{N}), 1112(\mathrm{C}-\mathrm{N})$, & $3.25(1 \mathrm{H}, \mathrm{dd}, \mathrm{HA}), 3.80(1 \mathrm{H}, \mathrm{dd}, \mathrm{H} \mathrm{B}), 5.50$ \\
& $706(\mathrm{C}-\mathrm{S})$. & $1 \mathrm{H}, \mathrm{dd}, \mathrm{H} \mathrm{X}), 6.80-7.50(11 \mathrm{H}, \mathrm{Ar}-\mathrm{H})$. \\
$\mathbf{2}$ & $1520(\mathrm{C}=\mathrm{N}), 1080(\mathrm{C}-\mathrm{N})$, & $3.10\left(6 \mathrm{H}, \mathrm{S}, \mathrm{N}\left(\mathrm{CH}_{3}\right)_{2}\right), 3.21(1 \mathrm{H}, \mathrm{dd}, \mathrm{HA})$, \\
& $690(\mathrm{C}-\mathrm{S})$. & $3.80(1 \mathrm{H}, \mathrm{dd}, \mathrm{HB}), 5.50(1 \mathrm{H}, \mathrm{dd}, \mathrm{HX}), 6.70-$ \\
& & $8.10(12 \mathrm{H}, \mathrm{Ar}-\mathrm{H})$. \\
$\mathbf{3}$ & $1595(\mathrm{C}=\mathrm{N}), 1097(\mathrm{C}-\mathrm{N})$, & $3.18(1 \mathrm{H}, \mathrm{dd}, \mathrm{HA}), 3.85(1 \mathrm{H}, \mathrm{dd}, \mathrm{HB}), 5.30$ \\
& $689(\mathrm{C}-\mathrm{S}), 822(\mathrm{C}-\mathrm{Cl})$. & $(1 \mathrm{H}, \mathrm{dd}, \mathrm{HX}), 6.40-7.90(12 \mathrm{H}, \mathrm{Ar}-\mathrm{H})$. \\
$\mathbf{4}$ & $1645(\mathrm{C}=\mathrm{N}), 1350(\mathrm{C}-\mathrm{N})$, & $3.05(1 \mathrm{H}, \mathrm{dd}, \mathrm{HA}), 3.91(1 \mathrm{H}, \mathrm{dd}, \mathrm{HB}), 5.6$ \\
& $716(\mathrm{C}-\mathrm{S}), 855(\mathrm{C}-\mathrm{Cl})$. & $(1 \mathrm{H}, \mathrm{dd}, \mathrm{HX}), 6.70-7.60(11 \mathrm{H}, \mathrm{Ar}-\mathrm{H})$. \\
$\mathbf{5}$ & $1640(\mathrm{C}=\mathrm{N}), 1352(\mathrm{C}-\mathrm{N})$, & $3.18(1 \mathrm{H}, \mathrm{dd}, \mathrm{HA}), 3.88(1 \mathrm{H}, \mathrm{dd}, \mathrm{HB}), 5.28$ \\
& $680(\mathrm{C}-\mathrm{S}), 8.70(\mathrm{C}-\mathrm{F})$. & $(1 \mathrm{H}, \mathrm{dd}, \mathrm{HX}), 6.80-7.45(12 \mathrm{H}, \mathrm{Ar}-\mathrm{H})$. \\
\hline
\end{tabular}




\section{Anti-inflammatory activity}

Synthesized compounds (1-5) were tested for their anti-inflammatory activity. Male albino rats weighing between 200-250 g were used for the experiment. Carragenan induced paw oedema method described by Singh and Ghosh $^{8}$ was followed for the acute antiinflammatory model and the results are presented in Table 3.

Table 3. Anti-inflammatory activity of the compounds (1-5).

\begin{tabular}{|c|c|c|c|c|c|c|}
\hline \multirow{2}{*}{ Compound } & \multicolumn{6}{|c|}{$\%$ increase in paw thickness of various time intervals } \\
\hline & $0.5 \mathrm{~h}$ & $01 \mathrm{~h}$ & $2 \mathrm{~h}$ & $3 \mathrm{~h}$ & $4 \mathrm{~h}$ & $6 \mathrm{~h}$ \\
\hline \multirow{2}{*}{ Standard } & 20.26 & 23.95 & 58.02 & 67.93 & 97.09 & 98.98 \\
\hline & \pm 0.64 & \pm 0.66 & \pm 1.54 & \pm 1.65 & \pm 1.95 & \pm 1.98 \\
\hline Control & $15.65 \pm$ & $21.95 \pm$ & $38.13 \pm$ & $43.15 \pm$ & $66.26 \pm$ & $75.85 \pm$ \\
\hline 1 & 1.42 & $2.30^{*}$ & 2.61 & $3.40^{*}$ & $3.96^{* *}$ & 1.53 \\
\hline \multirow[t]{2}{*}{2} & $20.62 \pm$ & $31.34 \pm$ & $46.38 \pm$ & $65.64 \pm$ & $71.63 \pm$ & $78.98 \pm$ \\
\hline & $2.57^{*}$ & $2.46^{*}$ & 1.52 & $3.85^{* *}$ & $2.85^{* * * *}$ & 1.87 \\
\hline \multirow[t]{2}{*}{3} & $18.84 \pm$ & $27.85 \pm$ & $42.85 \pm$ & $58.67 \pm$ & $79.7 \pm$ & $93.63 \pm$ \\
\hline & 1.52 & $3.42^{*}$ & $3.84^{* *}$ & 2.02 & 1.73 & 1.65 \\
\hline \multirow[t]{2}{*}{7} & $19.87 \pm$ & $31.48 \pm$ & $57.85 \pm$ & $62.12 \pm$ & $86.93 \pm$ & $96.4 \pm$ \\
\hline & $2.29^{*}$ & $3.65^{* *}$ & 2.42 & 1.45 & $3.14^{*}$ & 1.97 \\
\hline \multirow[t]{2}{*}{5} & $20.06 \pm$ & $31.69 \pm$ & $46.85 \pm$ & $58.84 \pm$ & $61.94 \pm$ & $92.75 \pm$ \\
\hline & 1.48 & $2.86^{*}$ & $3.76^{* *}$ & $1.75^{*}$ & $2.74^{* *}$ & 1.85 \\
\hline
\end{tabular}

Control: $1 \%$ sodium CMC gel, standard: aceclofenac standard and sample solution is $100 \mathrm{mg} / \mathrm{kg}$ body weight, values are expressed as mean $\pm(n=6) *=2.28, * *=3.75$, *** $=4.35 . P^{*}<0.05, P^{* *}$ $<0.01, P^{* * *}<0.001$ compared to control student $t$-test

\section{Results and Discussion}

The screening results revealed that the compounds (1-5) exhibited moderate to considerable activity when compared with reference standard aceclofenac. The synthesized compounds showed anti-inflammatory activity in the range of 50-80\% whereas standard drug showed $80-85 \%$ inhibition in paw edema. The results of anti-inflammatory activity indicated that compounds $\mathbf{3 , 4}$ and $\mathbf{5}$ showed maximum anti-inflammatory activity.

\section{Acknowledgment}

We are thankful to the Head, Sophisticated Instrumentation Facility, Indian Institute of Sciences, Bangalore for ${ }^{1} \mathrm{H}$ NMR spectra and to Sipra Laboratories, Hyderabad for IR spectra.

\section{References}

1. Edwards M L, Stemerick D M and Sunkara P S, J Med Chem., 1998, 33, 1948.

2. Rajendra Prasad Y, Ravi Kumar P, Asha Deepti Ch and Venkata Ramana M, Asian J Chem., 2007, 19(6), 4799.

3. Ankhiwala M D and Hathi M V, Indian J Heterocycl Chem., 1996, 5(3), 229.

4. Ballesteros J F, Sanz M J, Ubeda A, Miranda M A, Iborra S, Paya M and Alcaraz M J, J Med Chem., 1995, 38, 2794.

5. Bilgin A A, Palaska E, Sunal, Rumeysa and Gumesel B, Pharmazie, 1994, 49(1), 67.

6. Biligin A A, Palaska E and Sunal R, Arzneimittel-Forschung, 1993, 43(10), 1041.

7. Kumar M, Manoj P, Kumar, Ajay R and Ravi T K, Indian J Pharm Sci., 2005, 67(6), 755.

8. Singh H and Ghosh M N, J Pharm Pharmcol., 1958, 20, 316. 


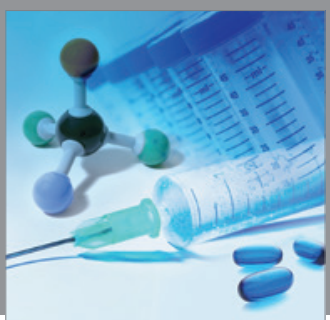

International Journal of

Medicinal Chemistry

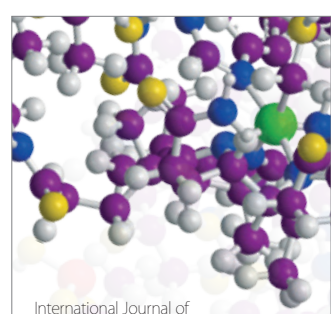

Carbohydrate Chemistry

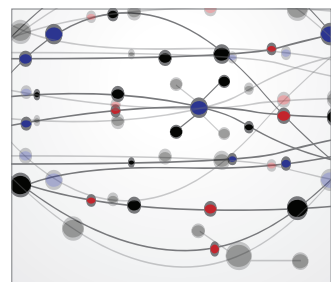

The Scientific World Journal
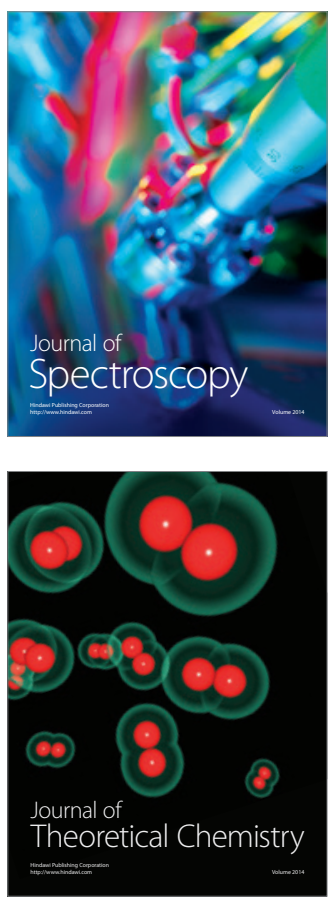
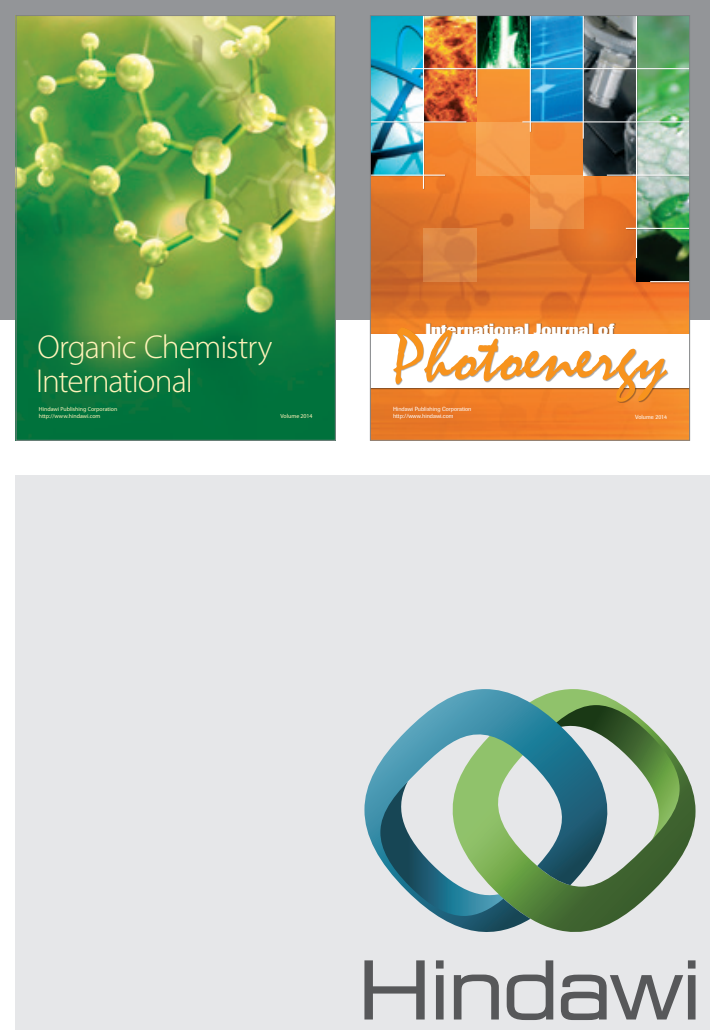

Submit your manuscripts at

http://www.hindawi.com
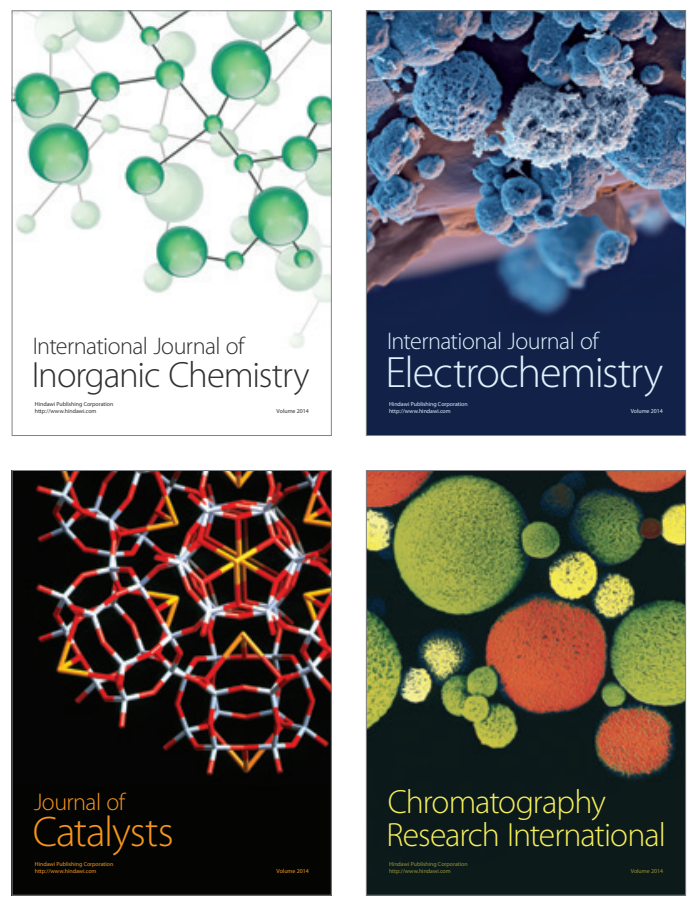
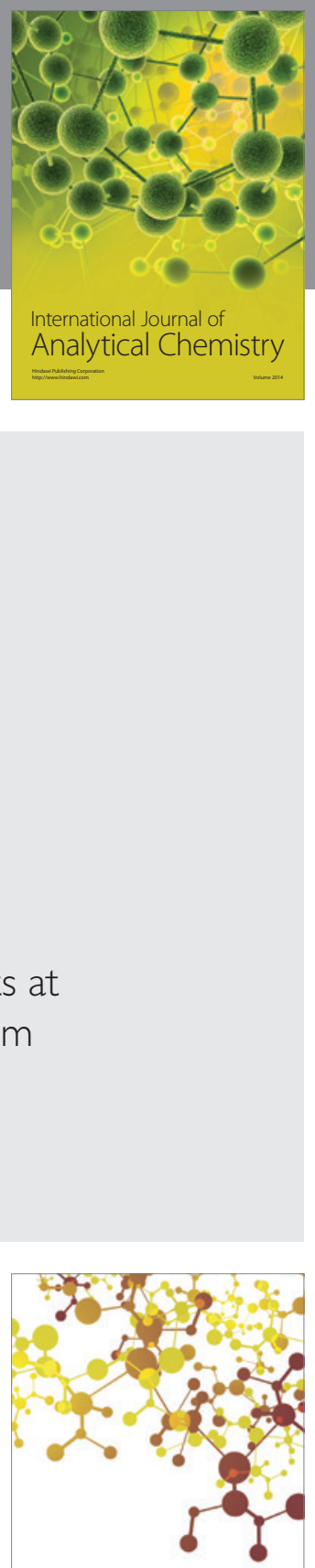

Journal of

Applied Chemistry
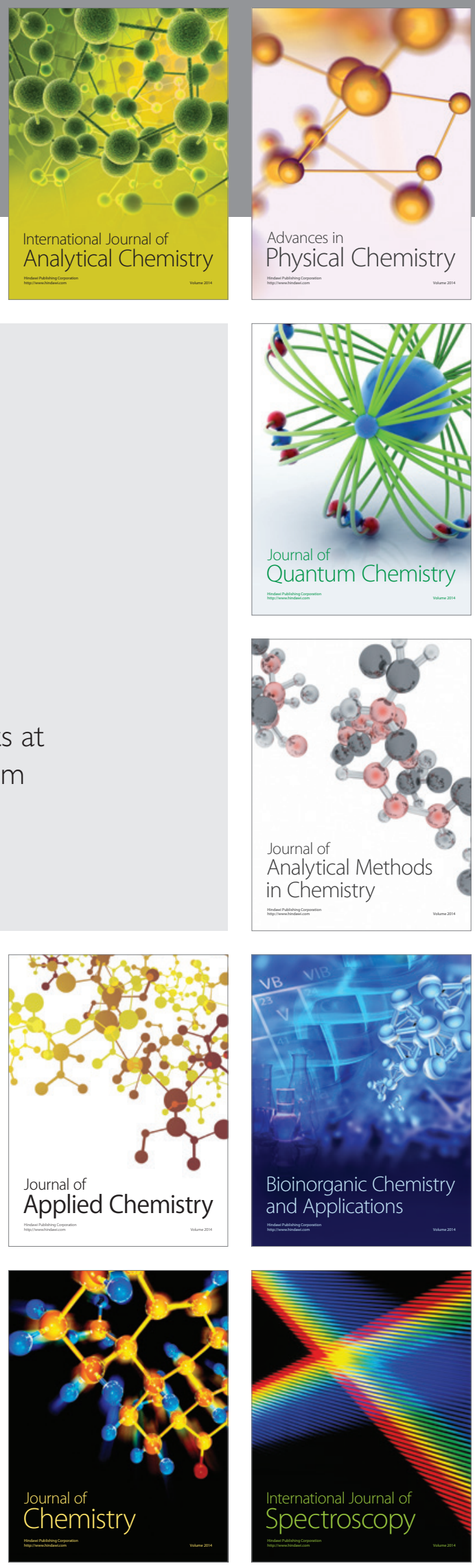